\title{
New trials for assessment of left atrial dysfunction by FDG-PET
}

\author{
Masanao Naya, MD, PhD, ${ }^{\text {a }}$ Osamu Manabe, $\mathrm{MD}, \mathrm{PhD}{ }^{\mathrm{b}}$ \\ and Nagara Tamaki, $M D, \mathrm{PhD}^{\mathrm{c}}$ \\ a Department of Cardiology, Hokkaido University, Sapporo, Japan \\ b Department of Nuclear Medicine, Hokkaido University, Sapporo, Japan \\ c Department of Radiology, Kyoto Prefectural University of Medicine, Kyoto, Japan
}

Received Sep 3, 2018; accepted Sep 4, 2018

doi: $10.1007 / \mathrm{s} 12350-018-01495-w$

\section{See related article, pp. 1547-1562}

The left atrium (LA) has an important role in modulating left ventricular filling and maintaining cardiac output. ${ }^{1}$ In addition, LA has been identified as an important biomarker of cardiovascular disease and adverse cardiovascular outcomes. ${ }^{2,3}$ Therefore, LA function has been focused for precisely and quantitatively analyzed recently. However, most of functional studies of LA have so far been done by echocardiography. ${ }^{4-6}$

Atrial fibrillation (AF) leads to progressive structural and functional changes in LA over time. LA fibrosis is a major determinant of the progression to, and burden of AF and its substrate, leading to an increase in total atrial activation time. ${ }^{7,8}$ A fibrosis burden demonstrated on late gadolinium contrast-enhanced cardiac magnetic resonance. ${ }^{9,10}$ There is evidence of progressive remodeling of the LA once AF develops with increasing fibrosis alongside lower LA reservoir strain in patients with persistent AF compared with patients with paroxysmal AF (PAF). ${ }^{9}$

PET has played an important role for functional and molecular imaging of the heart. FDG-PET has been used for identifying focal tumor lesions in atrial areas. ${ }^{10} \mathrm{With}$ improvement of image quality, PET has recently been used for assessing atrial tissue function. ${ }^{11,12}$ Of particular, FDG-PET has been used for assessing AF. With maximal use of high-resolution PET, it has a potential

Reprint requests: Nagara Tamaki, MD, PhD, Department of Radiology, Kyoto Prefectural University of Medicine, Kyoto, Japan; natamaki@med.hokudai.ac.jp

J Nucl Cardiol 2020;27:1563-65.

1071-3581/\$34.00

Copyright (C) 2019 American Society of Nuclear Cardiology. for identifying focus of AF as well as for assessing left atrial function quantitatively.

FDG is a glucose analog and has been used for tracking glucose metabolism. FDG-PET has two completely different roles for cardiovascular imaging. FDGPET has long been used for assessing tissue viability for patients with coronary artery disease. ${ }^{13}$ The heart derives its energy mainly from free fatty acids and glucose. FDG commonly enters cardiomyocytes through the glucose transporters 1 and 4. In ischemic state with the availability of glucose, FDG accumulation in the myocardium is maintained due to the dominant anaerobic glucose metabolism. On the other hand, FDG-PET has recently been used for identifying active inflammatory lesions because glucose is also consumed in the inflammatory process. ${ }^{14,15}$

Depending on the purposes of in vivo functional imaging, patient preparation should be carefully done before the FDG administration. ${ }^{16-18}$ Post-prandial condition, glucose loading, or insulin clamp is applied for myocardial viability assessment, whereas long fasting condition with or without heparin administration is required for identifying active inflammatory lesions with suppressing physiological myocardial FDG uptake.

The current paper includes retrospective clinical study to understand the feasibility of FDG-PET/CT under insulin clamp condition in order to relate scar and metabolic parameters identified by this method with structural and electrical remodeling across patients with varying degrees of AF burden in patients with sinus rhythm, PAF, and persistent AF. ${ }^{19}$ Ghannam and colleagues suggested that greater AF burden correlates with increased LA metabolism and scar. They have correlated the newly obtained metabolic activity with various structural parameters as well as ECG functional parameters. They suggested that those with AF may have greater LA volumes, increases in the amount and heterogeneity of LA metabolism, and greater amount of LA scar. 
It would be interesting to see potential mechanism to increase in FDG uptake in LA. If the increased metabolism of LA is secondary to AF in patients with HFrEF, what is the mechanism? Is hypometabolic scar a primary change to induce AF or a secondary change due to LA remodeling? Was this phenomenon due to partial volume effect as a result of increase in LA thickness or actual increase in glucose metabolism due to AF? The authors used both FDG and perfusion PET studies. What were the differences in perfusion and metabolism in LA areas in three different groups? It would be valuable to see whether AF may alter metabolism more than perfusion. The authors considered the inter-atrial septum to be part of the LA. But an elevated right atrial pressures may possibly influence FDG uptake in the inter-atrial septum. Thus, hemodynamic parameters may impact atrial metabolism. It would be nice to adjust for hemodynamic parameters during image acquisition.

Furthermore, some technical limitations should be discussed. There are significant partial volume effects for estimating metabolism as well as perfusion in thin wall, such as LA. Wall thickening in LA overload may significantly overestimate LA metabolism in $\mathrm{AF}$ as compared to sinus rhythm. In addition, ECG-gated acquisition may be required for precise assessment of tracer uptake in those with sinus vs AF. An image motion artifact may possibly underestimate metabolism and scar in AF as compared to sinus rhythm on gated acquisition. Second, delayed image (scan after 2-4 hours rather than 50 minutes) might improve the contrast between LA wall and LA cavity to detect slight uptake of the thin structure.

In order to validate the current unique and attractive findings, many more patient studies (preferably prospective study) using FDG-PET may be required. In addition, a precise comparison of ECG findings with various LA functional parameters is suggested using multi-imaging modalities, such as echocardiography, MRI, and PET perfusion and metabolic imaging. A high-resolution gadolinium-enhanced MRI (LGE) may hold a promise for precise assessment of LA scar and LA function simultaneously. On the other hand, FDGPET with Rb-82 perfusion can be done in any patients even after cardiac device installation. Moreover, PET may provide important information such as molecular and biochemical alterations in LA. Although the clinical importance to assess LA metabolism in addition to the size of LA is still unknown, hypometabolic scarring or its heterogeneity might predict patients with $\mathrm{AF}$ refractory to antiarrhythmic drugs and ablation therapy. In fact, $\mathrm{AF}$ is a significant predictor of heart failure hospitalization in patients with HFpEF. ${ }^{20}$ Potential mechanisms of LA remodeling and correlation with LA scar and regional dysfunction should fully be discussed. The current study raises the several interesting questions regarding LA metabolism in $\mathrm{AF}$ and heart failure.

\section{Disclosures}

The authors declare that there is no potential conflict of interest to disclose.

\section{References}

1. Matsuda Y, Toma Y, Ogawa H, et al. Importance of left atrial function in patients with myocardial infarction. Circulation 1983;67:566-71.

2. Abhayaratna WP, Seward JB, Appleton CP, et al. Left atrial size: Physiologic determinants and clinical applications. J Am Coll Cardiol 2006;47:2357-63.

3. Barbier P, Solomon SB, Schiller NB, et al. Left atrial relaxation and left ventricular systolic function determine left atrial reservoir function. Circulation 1999;100:427-36.

4. Hoit BD. Assessing atrial mechanical remodeling and its consequences. Circulation 2005;112:304-6.

5. Tops LF, Delgado V, Bertini M, et al. Left atrial strain predicts reverse remodeling after catheter ablation for atrial fibrillation. $\mathrm{J}$ Am Coll Cardiol 2011;57:324-31.

6. Hoit BD. Left atrial size and function: Role in prognosis. J Am Coll Cardiol 2014;63:493-505.

7. Platonov PG, Mitrofanova LB, Orshanskaya V, Ho SY. Structural abnormalities in atrial walls are associated with presence and persistency of atrial fibrillation but not with age. J Am Coll Cardiol 2011;58:2225-32.

8. Oakes RS, Badger TJ, Kholmovski EG, Akoum N, Burgon NS, Fish EN, Blauer JJ, Rao SN, DiBella EV, Segerson NM. Detection and quantification of left atrial structural remodeling with delayedenhancement magnetic resonance imaging in patients with atrial fibrillation. Circulation 2009;119:1758-67.

9. Kuppahally SS, Akoum N, Burgon NS, Badger TJ, Kholmovski EG, Vijayakumar S, Rao SN, Blauer J, Fish EN, Dibella EV, Macleod RS, McGann C, Litwin SE, Marrouche NF. Left atrial strain and strain rate in patients with paroxysmal and persistent atrial fibrillation: Relationship to left atrial structural remodeling detected by delayed-enhancement MRI. Circ Cardiovasc Imaging 2010;3:231-9.

10. Gheysens O, Cornillie J, Voigt JU, Bogaert J, Westhovens R. Left atrial myxoma on FDG-PET/CT. Clin Nucl Med 2013;38:e421-2.

11. Lange PS, Avramovic N, Frommeyer G, Wasmer K, Pott C, Eckardt L, Wenning C. Routine ${ }^{18}$ F-FDG PET/CT does not detect inflammation in the left atrium in patients with atrial fibrillation. Int J Cardiovasc Imaging 2017;33:1271-6.

12. Xie B, Chen BX, Wu JY, Liu X, Yang MF. Factors relevant to atrial ${ }^{18} \mathrm{~F}$-fluorodeoxyglucose uptake in atrial fibrillation. J Nucl Cardiol 2018. https://doi.org/10.1007/s12350-018-1387-4.

13. Ghosh N, Rimoldi OE, Beanlands RSB, Camici PG. Assessment of myocardial ischaemia and viability: Role of positron emission tomography. Eur Heart J 2010;31:2984-95.

14. Juneau D, Erthal F, Alzahrani A, Alenazy A, Nery PB, Beanlands RS, Chow BJ. Systemic and inflammatory disorders involving the heart: The role of PET imaging. Q J Nucl Med Mol Imaging 2016;60:383-96.

15. Nensa F, Kloth J, Tezgah E, Poeppel TD, Heusch P, Goebel J, Nassenstein K, Schlosser T. Feasibility of FDG-PET in 
myocarditis: Comparison to CMR using integrated PET/MRI. J Nucl Cardiol 2018;25:785-94.

16. Tamaki N, Yonekura Y, Konishi J. Myocardial FDG PET studies with the fasting, oral glucose-loading or insulin clamp methods. J Nucl Med 1992;33:1263, 1266-8.

17. Scholtens AM, Verberne HJ, Budde RP, Lam MG. Additional heparin preadministration improves cardiac glucose metabolism suppression over low-carbohydrate diet alone in ${ }^{18}$ F-FDG PET imaging. J Nucl Med 2016;57:568-73.

18. Manabe O, Yoshinaga K, Ohira H, Masuda A, Sato T, Tsujino I, Yamada A, Oyama-Manabe N, Hirata K, Nishimura M, Tamaki N. The effects of 18-h fasting with low-carbohydrate diet preparation on suppressed physiological myocardial ${ }^{(18)}$ F-fluorodeoxyglucose (FDG) uptake and possible minimal effects of unfractionated heparin use in patients with suspected cardiac involvement sarcoidosis. J Nucl Cardiol 2016;23:244-52.

19. Ghannam M, Yun HJ, Ficaro EP, et al. Multiparametric assessment of left atrial remodeling using ${ }^{18} \mathrm{~F}$-FDG PET/CT cardiac imaging: A pilot study. J Nucl Cardiol 2018. https://doi.org/10. 1007/s12350-018-1429-y.

20. Cikes M, Claggett B, Shah AM, et al. Atrial fibrillation in heart failure with preserved ejection fraction: The TOPCAT trial. JACC Heart Fail 2018;6:689-97. 